\title{
Теория упругости
}

УДК 539.375

DOI: $10.22363 / 1815-5235-2018-14-3-248-257$

\section{МОДЕЛИРОВАНИЕ ТРЕЩИНООБРАЗОВАНИЯ В ВОЛОКНИСТОМ КОМПОЗИТЕ ПРИ ИЗГИБЕ}

\author{
Ш.Г. ГАСАНОВ \\ Азербайджанский технический университет \\ пр. Г. Джавида, д. 25, Баку, Азербайджан, AZ 1073 \\ (поступила в редакц̧ию: 4 января 2018 г.; принята к публикацчии: 30 апреля 2018 г.)
}

\begin{abstract}
Проектирование армированного волокнами композита минимальной материалоемкости при гарантированной надежности и долговечности требует учета случаев, когда в связующем могут возникать трещины. Чтобы знать предельные изгибающие нагрузки, при которых в связующем произойдет образование трещин, необходимо проводить предельный анализ композита. На основе предложенной расчетной модели, учитывающей в волокнистом композите наличие повреждений (зон ослабленных межчастичных связей материала), разработан метод расчета параметров композита, при которых появляются трещины. Рассмотрена тонкая пластина из упругой изотропной среды (матрицы) и распределенных в ней включений (волокон) из другого упругого материала при изгибе. Считается, что при нагружении происходит зарождение трещин и разрушение композита. Для прогнозирования появления трещин в волокнистом композите при изгибе в зависимости от геометрических и механических характеристик связующего и волокна построена замкнутая система нелинейных алгебраических уравнений. Сформулирован критерий зарождения трещин в композите при действии изгибающих нагрузок. Размер предельных минимальных зон ослабленных межчастичных связей материала, при которых происходит трещинообразование, рекомендуется рассматривать как проектную характеристику материала связующего.
\end{abstract}

Ключевые слова: связующее, волокно, изгиб, зоны предразрушения, композитная пластина, трещинообразование

\section{MODELING OF CRACKS NUCLEATION IN FIBER COMPOSITE UNDER BENDING}

\author{
SH.H. HASANOV \\ Azerbaijan Technical University \\ 25 H. Javid Ave, Baku, AZ 1073, Azerbaijan \\ (received: January 04, 2018; accepted: April 30,2018)
}

\begin{abstract}
Design of fiber-reinforced composite of minimum material consumption at guaranteed reliability and durability requires consideration of cases when cracks may appear in the binder. To know the limit bending loads at which cracks will occur in the binder, it is necessary to carry out the limit analysis of the composite. Proposed design model takes into account the presence of damages (zones of weakened inter-particle bonds of the material) in the fiber composite. Based on this design model a calculation method has been developed for composite parameters at which cracks appear. A thin plate of elastic isotropic medium (matrix) and inclusions (fibers) of another elastic material distributed in the matrix is considered. The plate is bending. It is assumed that at the loading of composite, the cracks initiation and fracture of the composite occur. A closed system of nonlinear algebraic equations is constructed. Solution of the obtained system allows to predict the cracking in composite under bending, depending on geometric and mechanical characteristics of the binder and fiber. A criterion of the cracks nucleation in the composite under the action of bending loads is formulated. Size of limit minimal zones of weakened inter-particle bonds of the material at which the cracks nucleation occurs is recommended to be considered as a design characteristic of the binder material.
\end{abstract}

Keywords: binder, fiber, bending, prefracture zones, composite plate, cracking 
Введение. Создание новых композиционных материалов, обладающих высокой прочностью, жесткостью и надежностью, открывает большие возможности их широкого применения в различных областях строительства и машиностроения. Такими материалами, в частности, являются волокнистые композиты.

Решение различных задач техники требует объективной информации о напряженно-деформированном состоянии в элементах конструкций из композиционных материалов. Эту информацию можно получить только при учете основных особенностей этих материалов. При проектировании новых конструкций из композитных материалов необходимо учитывать возможность появления в материале трещин. Поэтому необходимо проводить предельный анализ, чтобы установить, что предполагаемые исходные повреждения, расположенные максимально неблагоприятно, не будут расти до критических размеров и не вызовут разрушения в течение расчетного срока службы. Одно из основных мест в механике композитных материалов занимают проблемы, связанные с особенностями из структуры. В частности, при исследовании различного рода вопросов механики составных тел важно учитывать повреждения в их структурах. Такие повреждения могут быть вызваны самим построением композитных материалов, технологическими процессами или действием различных факторов. Следует отметить, что успешное применение на практике композитных материалов в значительной мере связано с решением задач определения их напряженно-деформированного состояния с учетом структурных особенностей, в том числе повреждений в связующем и волокнах. Поэтому исследования напряженно-деформированного состояния в волокнистых композитах с повреждениями следует признать весьма актуальными. Этим вопросам посвящено большое число работ [1-27] и др. Разработка математической модели, позволяющей прогнозировать напряженно-деформированное состояние композита в стадии предразрушения (образования трещин), имеет большое значение.

Цель работы состоит в разработке расчетной модели для составного тела «связующее - волокно», позволяющей рассчитать предельные внешние изгибающее нагрузки, при которых происходит образование трещин в волокнистом композите.

Постановка задачи. Пусть неограниченная составная пластина (волокнистый композит) подвергается изгибу средними моментами (изгиб на бесконечности) $M_{x}=M_{x}^{\infty}, M_{y}=M_{y}^{\infty}, H_{x y}=0$.
При нагружении композита в материале связующего будут возникать зоны предразрушения. Зоны предразрушения моделируются как области ослабленных межчастичных связей материала, где при нагружении композита имеет место пластическое течение. Зоны предразрушения ориентированы в направлении максимальных растягивающих напряжений. Исследования [28-30] показывают, что в начальной стадии нагружений зоны предразрушения представляют собой узкий вытянутый слой, а затем с ростом внешней нагрузки внезапно появляется вторичная система зон ослабленных межчастичных связей материала.

Пусть внешняя изгибающая нагрузка изменяется таким образом, что в зонах ослабленных межчастичных связей материала связующего осуществляется пластическое деформирование. После некоторого числа циклов нагружения композита возможность пластического деформирования в зонах ослабленных межчастичных связей материала исчерпывается и резко возрастает раскрытие берегов зон пластического течения. Когда раскрытие берегов зоны предразрушения в точке максимальной концентрации достигнет предельного значения $\delta_{c}$ для данного материала связующего, в этой точке образуется трещина (произойдет разрыв межчастичных связей материала) [31].

$\mathrm{B}$ процессе нагружения композита изгибающими моментами в материале связующего будет возникать зона предразрушения. Для математического описания взаимодействия берегов зоны предразрущения принято, что в этой зоне между берегами имеются связи, которые сдерживают раскрытие берегов зоны ослабленных межчастичных связей материала. Взаимодействие берегов зоны предразрушения моделируется с помощью введения между берегами линий пластического скольжения (вырожденных полос пластических деформаций). Местоположение и размеры зон пластического течения зависят от вида материала связующего и нагружения. Считается, что в зонах предразрущения имеет место пластическое течение при постоянном напряжении. При этом местоположение и размер зон предразрушения изначально неизвестны и должны быть определены в процессе решения задачи.

Анализ взаимодействия связующего и волокон проводится на основе модели с одним волокном. Остальные волокна «размазываются», а материал вне выделенного волокна принимается однородным и изотропным с соответствующими эффективными упругими постоянными (по правилу смесей). Взаимодействие размазанных волокон и зон предразрушения осуществляется через соот- 
ветствующие эффективные упругие постоянные. При этом нет ограничений на расположение и относительные размеры волокон и зон предразрушения, но принято, что зоны предразрушения не пересекаются между собой и волокном.

Начало системы координат $O x y$ совпадает с геометрическим центром волокна в срединной плоскости композитной пластины (рис. 1). В круговое отверстие связующего вставлено волокно из другого, также упругого материала. Принято, что всюду на границе соединения $L(\tau=R \exp (i \theta))$ имеет место жесткое сцепление различных материалов.

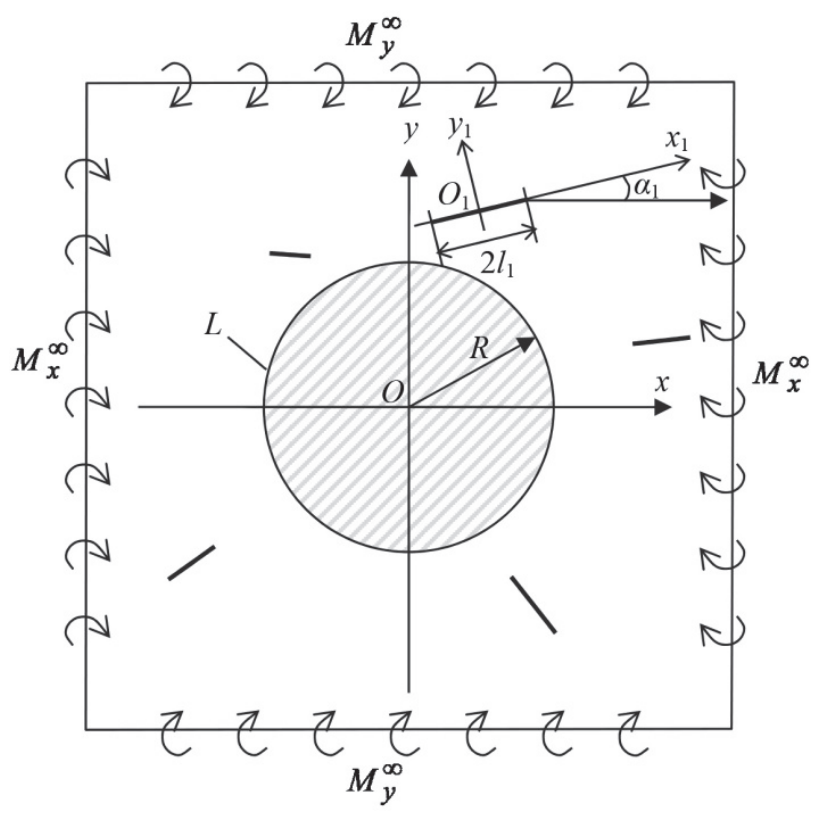

Рис. 1. Расчетная схема образования трещин в связующем композита при изгибе

[Fig. 1. Design diagram of cracks nucleation in the composite binder subject to bending]

Рассмотрим случай, когда в связующем вблизи волокна в процессе изгиба имеется $N$ прямолинейных зон предразрушения длиной $2 l_{k}$ $(k=1,2, \ldots, N)$. В центрах прямолинейных зон предразрушений разместим начала локальных систем координат $O_{k} x_{k} y_{k}$. Оси $x_{k}$ совпадают с линиями зон предразрушения и составляют осью $x$ углы $\alpha_{k}$. На контуре раздела сред должны выполняться условия:

$$
\begin{gathered}
w=w_{0}, \frac{\partial w}{\partial n}=\frac{\partial w_{0}}{\partial n}, \frac{\partial w}{\partial t}=\frac{\partial w_{0}}{\partial t}, \\
\frac{\partial^{2} w}{\partial n^{2}}=\frac{\partial^{2} w_{0}}{\partial n^{2}}, \frac{\partial^{2} w}{\partial t^{2}}=\frac{\partial^{2} w_{0}}{\partial t^{2}}, \frac{\partial^{2} w}{\partial n \partial t}=\frac{\partial^{2} w_{0}}{\partial n \partial t},
\end{gathered}
$$

где $w$ и $w_{0}$ прогибы связующего и волокна соответственно; $n$ и $t$ натуральные координаты (нормаль и касательная к контуру $L$ ).
Соотношения (1) являются следствием непрерывности прогибов в композите, углов наклона касательной и величин изгибающих моментов.

При нагружении композита внешними изгибающими моментами в связях, соединяющих берега зон предразрушения, будут возникать нормальные $\sigma_{y_{k}}=\sigma_{s}$ и касательные напряжения $\tau_{x_{k} y_{k}}=\tau_{s}\left(\sigma_{s}-\right.$ предел текучести материала при растяжении, $\tau_{s}$ - предел текучести материала на сдвиг).

Граничные условия на берегах зон предразрушения будут иметь вид:

$$
M_{n}=M_{s}, N_{n}+\frac{\partial H_{n t}}{\partial t}=H_{s},
$$

где $M_{s}=\frac{\sigma_{s} h^{2}}{4} ; H_{s}=\frac{\tau_{s} h^{2}}{4} ; h-$ толщина композитной пластины; $M_{n}, H_{n t}$ - удельные изгибающий и крутящий моменты; $N_{n}$ - удельная поперечная сила.

Для определения значений внешней изгибающей нагрузки, при которой произойдет появление трещин в связующем, необходимо постановку задачи дополнить условием трещинообразования (разрыва межчастичных связей материала). В качестве такого условия принимается критерий предельного раскрытия берегов зоны ослабленных межчастичных связей материала:

$$
\left|\left(v_{k}^{+}-v_{k}^{-}\right)-i\left(u_{k}^{+}-u_{k}^{-}\right)\right|=\delta_{c}(k=1,2, \ldots, N) .
$$

Здесь $\delta_{c}$-характеристика сопротивления материала связующего трещинообразованию; $\left(v_{k}^{+}-v_{k}^{-}\right)-$ нормальная составляющая раскрытия берегов $k$-той зоны предразрушения; $\left(u_{k}^{+}-u_{k}^{-}\right)-$касательная составляющая раскрытия (сдвига) берегов $k$-той зоны предразрушения.

Дополнительное условие (3) позволяет установить параметры композитной пластины, при которых произойдет появление трещин в связующем.

Моменты $M_{x}, M_{y}, H_{x y}$, поперечные силы $N_{x}$, $N_{y}$ и прогиб $w$ в технической теории изгиба пластин можно представить с помощью комплексных потенциалов Колосова - Мусхелишвили [32]. На границе раздела сред имеем

$$
\begin{gathered}
\varphi(\tau)+\tau \overline{\Phi(\tau)}+\overline{\psi(\tau)}=\varphi_{0}(\tau)+\tau \overline{\Phi_{0}(\tau)}+\overline{\psi_{0}(\tau)}, \\
n_{*} \varphi(\tau)+\tau \overline{\Phi(\tau)}+\overline{\psi(\tau)}= \\
=\frac{D_{0}\left(1-v_{0}\right)}{D(1-v)}\left\{n_{0} \varphi_{0}(\tau)+\tau \overline{\Phi_{0}(\tau)}+\overline{\psi_{0}(\tau)}\right\} .
\end{gathered}
$$


Здесь $\varphi(\tau), \psi(\tau)$ и $\varphi_{0}(\tau), \psi_{0}(\tau)$ - комплексные потенциалы для связующего и волокна соответственно; $\tau=\exp (i \theta)$ - переменная точка на границе раздела сред; $n_{*}=-(3+v) /(1-v)$; $D$ и $D_{0}-$ цилиндрическая жесткость связующего и волокна соответственно; $v$ и $v_{0}$ - коэффициенты Пуассона материала связующего и волокна; $n_{0}=-\left(3+v_{0}\right) /\left(1-v_{0}\right)$.

Рассматриваемая краевая задача механики композитных материалов оказывается задачей теории упругости с неизвестной границей и ее требуется определить в процессе решения краевой задачи. На берегах прямолинейных зон предразрушения имеем следующие условия

$$
\begin{gathered}
n_{*} \Phi\left(x_{k}\right)+\overline{\Phi\left(x_{k}\right)}+x_{k} \overline{\Phi^{\prime}\left(x_{k}\right)}+\overline{\Psi\left(x_{k}\right)}=f_{k}^{0}+i C_{k} \\
(k=1,2, \ldots, N),
\end{gathered}
$$

где $f_{k}^{0}=M_{s}-i H_{s} ; x_{k}-$ аффикс точек $k$-той зоны предразрушения; $C_{k}$-действительные постоянные, определяемые в ходе решения задачи из условий равенства нулю скачка прогиба в вершинах зон предразрушения.

В принятых предположениях теории Кирхгофа рассматриваемая задача определения напряженно-деформированного состояния композитной пластины сводится к отысканию двух пар функций $\Phi_{0}(z), \Psi_{0}(z)$ и $\Phi(z), \Psi(z)$ комплексной переменной $z=x+i y$, аналитических в соответствующих областях и удовлетворяющих краевым условиям (4)-(6).

Комплексные потенциалы $\varphi_{0}(\tau)$ и $\psi_{0}(\tau)$, описывающие напряженно-деформированное состояние волокна, ищем в виде

$$
\varphi_{0}(z)=\sum_{k=1}^{\infty} a_{k} z^{k}, \quad \Psi_{0}(z)=\sum_{k=1}^{b} b_{k} z^{k} .
$$

Обозначим левую часть краевого условия (4) как $f_{1}+i f_{2}$ и примем, что на контуре $L$ эта комплексная функция разлагается в ряд Фурье:

$$
f_{1}+i f_{2}=\sum_{k=-\infty}^{\infty} A_{k} e^{i n \theta} .
$$

На основании краевого условия (4) и соотношений (7), (8), используя метод степенных рядов [32], находим коэффициенты $a_{n}, b_{n}$ функций $\varphi_{0}(z)$ и $\psi_{0}(z)$ :

$$
\begin{aligned}
& a_{n}=\frac{A_{n}}{R^{n}} \quad(n>1), \quad \operatorname{Re} a_{1}=\frac{A_{1}}{2 R}, \\
& b_{n}=\frac{\bar{A}_{-n}}{R^{n}}-(n+2) \frac{A_{n+2}}{R^{n}} \quad(n \geq 0) .
\end{aligned}
$$

Величины коэффициентов $A_{n}$ ищем в ходе решения задачи для связующего. С помощью комплексных потенциалов $\varphi_{0}(z)$ и $\psi_{0}(z)$ после некоторых элементарных преобразований граничные условия на контуре $\tau=R \exp (i \theta)$ раздела сред запишем в виде

$$
\begin{gathered}
\varphi(\tau)+\tau \overline{\Phi(\tau)}+\overline{\psi(\tau)}=\sum_{k=-\infty}^{\infty} A_{k} e^{i k \theta}, \\
n_{*} \varphi(\tau)+\tau \overline{\Phi(\tau)}+\overline{\psi(\tau)}= \\
=\frac{D_{0}\left(1-v_{0}\right)}{D(1-v)}\left\{n_{0} \sum_{k=1}^{\infty} a_{k} R^{k} e^{i k \theta}+\bar{a}_{1} \operatorname{Re}^{i \theta}+\right. \\
\left.+\sum_{k=0}^{\infty}(k+2) \bar{a}_{k+2} R^{2 k+2} e^{-i k \theta}+\sum_{k=0}^{\infty} \bar{b}_{k} R^{k} e^{-i k \theta}\right\} .
\end{gathered}
$$

Решение граничной задачи (6), (10), (11) ищем в виде

$$
\begin{gathered}
\Phi(z)=\varphi_{1}^{\prime}(z)+\Phi_{2}(z), \quad \Psi(z)=\psi_{1}^{\prime}(z)+\Psi_{2}(z), \\
\varphi_{1}(z)=-\frac{M_{x}^{\infty}+M_{y}^{\infty}}{4(1+v) D} z+\sum_{k=1}^{\infty} c_{k} z^{-k} \\
\Psi_{1}(z)=\frac{M_{y}^{\infty}-M_{x}^{\infty}}{2(1+v) D} z+\sum_{k=1}^{\infty} d_{k} z^{-k} \\
\Phi_{2}(z)=\frac{1}{2 \pi i(1+\kappa)} \sum_{k=1}^{N} \int_{-l_{k}}^{l_{k}} \frac{g_{k}(t)}{t-z_{k}} d t \\
=\frac{1}{2 \pi i(1+\kappa)} \sum_{k=1}^{N} e^{-2 i \alpha_{k}} \int_{-l_{k}}^{l_{k}}\left[\frac{\kappa(z)}{\operatorname{\kappa g}(t)}-\frac{\bar{T}_{k} e^{i \alpha_{k}}}{\left(t-z_{k}\right)^{2}} g_{1}(t)\right] d t
\end{gathered}
$$

где $T_{k}=t e^{i \alpha_{k}}+z_{k}^{0} ; \quad z_{k}=e^{-i \alpha_{k}}\left(z-z_{k}^{0}\right) ; \quad \kappa=(3-v) /(1+v) ;$ $g_{k}\left(x_{k}\right)$ - искомые функции, характеризующие разрыв углов поворота срединной плоскости пластины при переходе через линию зоны предразрушения

$$
\pm g_{k}(t)=\frac{d}{d t}\left(\frac{\partial W_{k}^{ \pm}}{\partial x_{k}}+i \frac{\partial W_{k}^{ \pm}}{\partial y_{k}}\right) .
$$

Удовлетворяя функциями (12), (13) и (14) граничным условиям (10), (11) и сравнивая коэффициенты при одинаковых степенях $\exp (i \theta)$, получим алгебраические уравнения для нахождения коэффициентов $c_{k}, d_{k}$ и $A_{k}$. Эти соотношения позволяют получить формулы для $c_{k}, d_{k}$ и $A_{k}$ в явном виде через функции $g_{k}\left(x_{k}\right)$.

Удовлетворяя краевым условиям на берегах зон предразрушения (6), получим систему $N$ комплексных сингулярных интегральных урав- 
нений относительно неизвестных функций $g_{k}\left(x_{k}\right)$ $(k=1,2, \ldots, N)$ :

$$
\begin{gathered}
\sum_{k=1}^{N} \int_{-l_{k}}^{l_{k}}\left[R_{n k}(t, x) g_{n}^{*}(t)+S_{n k}(t, x) \overline{g_{n}^{*}(t)}\right] d t=\pi F_{n}(x), \\
|x| \leq l_{n}(n=1,2, \ldots, N),
\end{gathered}
$$

где $l_{n}, t, x$ и $z_{n}^{0}$ - безразмерные величины, отнесенные к $R ; g_{k}^{*}(t)=\frac{g_{k}(t)}{i(1+\kappa)} ; R_{n k}, S_{n k}$ определяются по известным соотношениям [33, формулы VI. 62];

$$
\begin{gathered}
F_{n}(x)=f_{n}^{0}+i C_{n}- \\
-\left[n_{*} \Phi_{1}\left(x_{n}\right)+\overline{\Phi_{1}\left(x_{n}\right)}+x_{n} \overline{\Phi_{1}^{\prime}\left(x_{n}\right)}+\overline{\Psi_{1}\left(x_{n}\right)}\right] .
\end{gathered}
$$

К системе сингулярных интегральных уравнений (16) для внутренних зон предразрушения добавляются равенства, которые обеспечивают однозначность углов поворота срединной плоскости композитной пластины при обходе контуров зон предразрушения

$$
\int_{-l_{k}}^{l_{k}} g_{k}^{*}(t) d t=0 \quad(k=1,2, \ldots, N) .
$$

Для определения постоянных $C_{k}(k=1,2, \ldots, N)$ имеем соотношения [34], обеспечивающие равенство нулю скачка прогиба в вершинах зон предразрушения

$$
\operatorname{Re} \int_{-l_{k}}^{l_{k}} \operatorname{tg}_{k}(t) d t=0 \quad(k=1,2, \ldots, N)
$$

Система комплексных сингулярных интегральных уравнений (16) при дополнительных условиях (17) сводится [28; 33] к конечной системе $N \times M$ алгебраических уравнений относительно приближенных значений искомых функций $g_{k}^{*}\left(x_{k}\right)$ в узловых точках:

$$
\begin{gathered}
\frac{1}{M} \sum_{k=1}^{M} \sum_{m=1}^{N} l_{k}\left[g_{k}^{*}\left(t_{m}\right) R_{n k}\left(l_{k} t_{m}, l_{n}, x_{n}\right)+\overline{g_{k}^{*}\left(t_{m}\right)} S_{n k}\left(l_{k} t_{m}, l_{n}, x_{n}\right)\right]= \\
=F_{n}\left(x_{r}\right), \\
\sum_{m=1}^{M} g_{n}^{*}\left(t_{m}\right)=0 \quad(r=1,2, \ldots, M-1 ; n=1,2, \ldots, N) .
\end{gathered}
$$

Если в системе (19) перейти к комплексно сопряженным величинам, то получим еще одну систему $N \times M$ алгебраических уравнений.

Решение системы сингулярных интегральных уравнений ищется в классе всюду ограниченных функций (напряжений). Поэтому к системе (19) необходимо добавить условия ограниченности напряжений на концах зон пластического течения $x_{k}= \pm l_{k}(k=1,2, \ldots, N)$. Эти $2 N$ условий имеют следующий вид

$$
\begin{gathered}
\sum_{m=1}^{M}(-1)^{M+m} g_{k}^{*}\left(t_{m}\right) \operatorname{tg} \frac{2 m-1}{4 M} \pi=0 \quad(k=1,2, \ldots, N), \\
\sum_{m=1}^{M}(-1)^{m} g_{k}^{*}\left(t_{m}\right) \operatorname{ctg} \frac{2 m-1}{4 M} \pi=0 .
\end{gathered}
$$

$2 N$ комплексных уравнений (20) служат для определения координат вершин зон пластического течения. Алгебраическая система (19), (20) изза неизвестных размеров зон предразрушения является нелинейной. Полученные разрешающие системы уравнений относительно $c_{k}, d_{k}, A_{k}$, $g_{k}^{*}\left(t_{m}\right) \quad(k=1,2, \ldots, N ; m=1,2, \ldots, M)$ позволяют для заданной внешней изгибающей нагрузки исследовать напряженно-деформированное состояние композита при наличии в материале связующего произвольного числа зон предразрушения. Для решения нелинейной объединенной алгебраической системы используется метод последовательных приближений. Ее численное решение позволяет найти координаты вершин (местоположение) и размеры зон предразрушения, значения $c_{k}, d_{k}, A_{k}, g_{k}^{*}\left(t_{m}\right)$. Очевидно, что, определив координаты вершин всех зон предразрушения, по известным формулам аналитической геометрии можно найти координаты $z_{k}^{0}$ центров зон предразрушения и углы $\alpha_{k}$ с осью $x$ (рис. 1). После нахождения значений искомых функций $g_{k}^{*}\left(t_{m}\right)$ вычислялось раскрытие берегов зон предразрушения. С помощью критерия предельного раскрытия берегов зон предразрушения найдено условие, определяющее критический уровень внешней изгибающей нагрузки в связующем для каждой зоны предразрушения. Значение внешней изгибающей нагрузки, вызывающей появление трещины в $k$-той зоне предразрушения, определяется из следующего соотношения

$$
\left|-\int_{-l_{k}}^{x_{k}^{0}} g_{k}^{*}\left(x_{k}\right) d x_{k}\right|=\delta_{c} \quad(k=1,2, \ldots, N),
$$

где $x_{k}^{0}$ - координаты точки зон предразрушения, в которых происходит разрыв межчастичных связей материала связующего.

Критическим значением изгибающей нагрузки в связующем будет минимальное среди величин, определяемых соотношением (21).

Результаты расчетов приведены на рис. 2. 


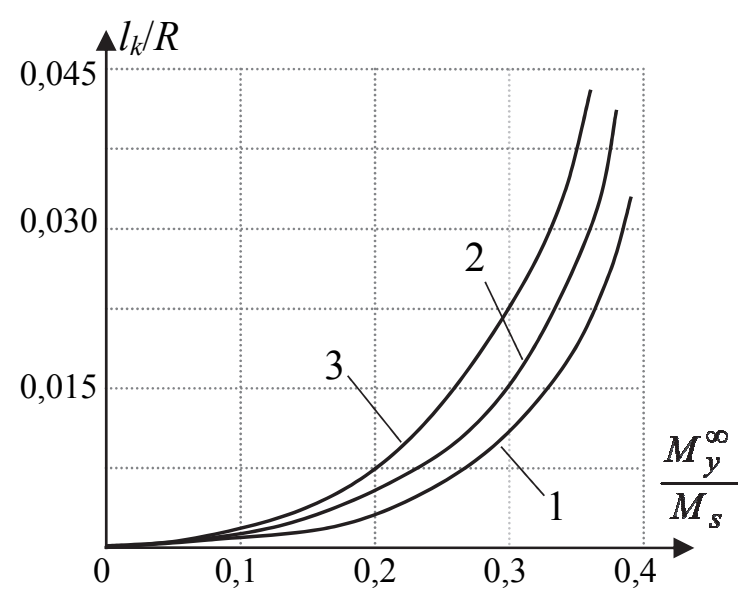

Рис. 2. Зависимость длин зон предразрушения от внешней изгибающей нагрузки

[Fig. 2. Dependence of the prefracture zones lengths on external bending load]
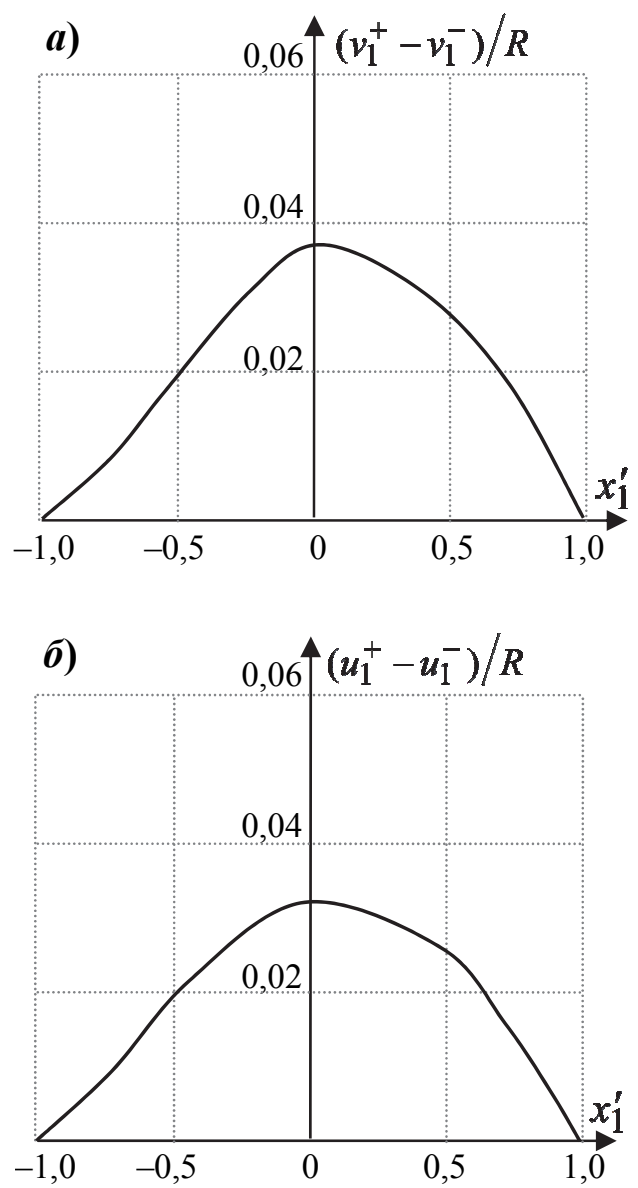

Рис. 3. Распределение нормальных (a) и касательных (б) оставляющих вектора перемещений для угла ориентации $\alpha_{1}=11^{\circ}$

[Fig. 3. Distribution of the normal (a) and tangential ( $\sigma$ ) components of displacement vector for orientation angle $\alpha_{1}=11^{\circ}$ ]

На рис. 2 представлены зависимости длины зоны предразрушения $l_{k} / R(k=1,2,3)$ от безраз- мерного значения изгибающего момента $M_{y}^{\infty} / M_{s}$ для различных углов ориентации $\left(\alpha_{1}=11^{\circ}, \alpha_{2}=36^{\circ}\right.$, $\alpha_{3}=48^{\circ}$ ). В расчетах было принято $M=30$.
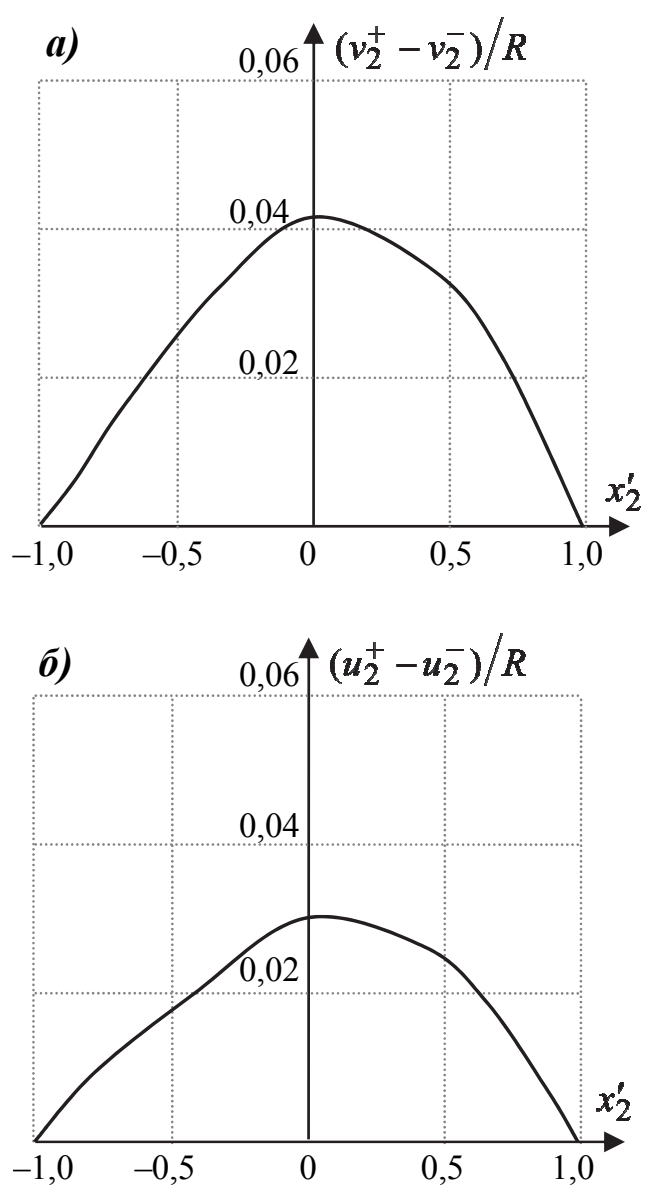

Рис. 4. Распределение нормальных (a) и касательных (б) составляющих вектора перемещений для угла ориентации $\alpha_{1}=36^{\circ}$

[Fig. 4. Distribution of the normal (a) and tangential (ס) components of displacement vector for orientation angle $\alpha_{1}=36^{\circ}$ ]

На рис. 3-5 представлены графики распределения нормальных $\left(v_{k}^{+}-v_{k}^{-}\right) / R$ и касательных $\left(u_{k}^{+}-u_{k}^{-}\right) / R$ составляющих вектора перемещений. При расчетах были использованы безразмерные координаты $x_{k}^{\prime}=x_{k} / l_{k}$.

Существенное влияние на раскрытие берегов зон пластического течения играет местоположение зон предразрушения. Когда зоны пластического течения расположены близко друг к другу, то расчеты показывают как увеличение размеров зон предразрушения и раскрытие их берегов, так и уменьшение раскрытия берегов и размеров зон предразрушения. Различие видов вза- 
имного влияния повреждений (зон ослабленных межчастичных связей материалов) объясняется различиями их расположения.

Полученная объединенная алгебраическая система уравнений задачи позволяет получить решение с любой наперед заданной точностью.
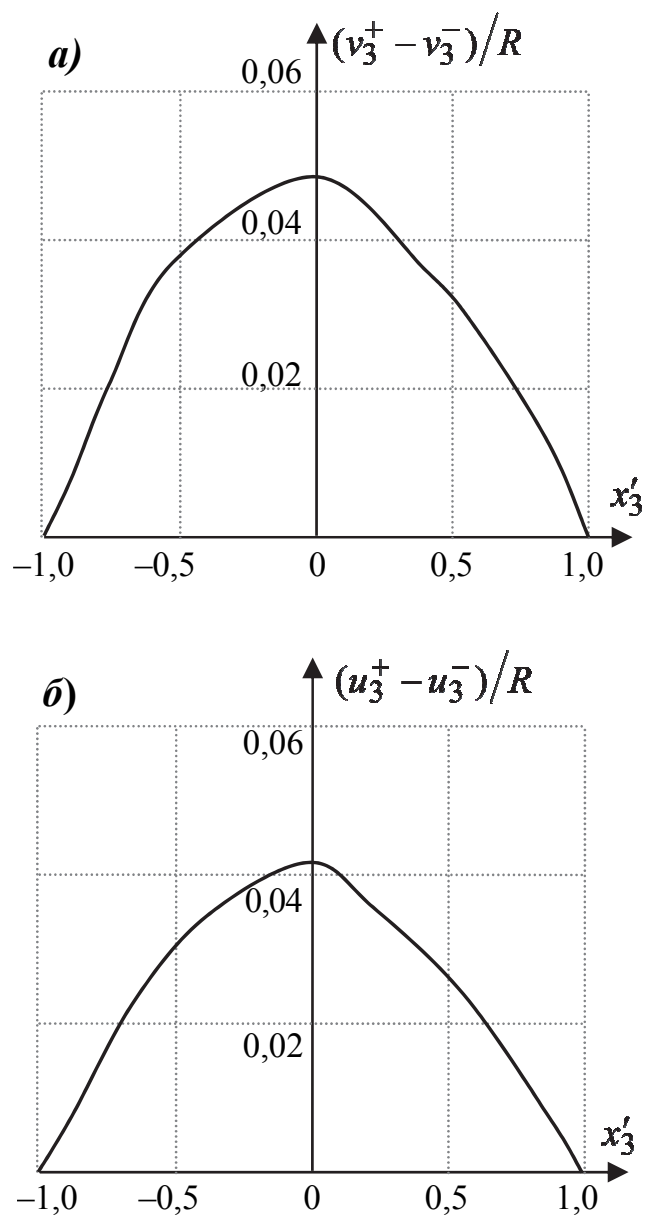

Рис. 5. Распределение нормальных (а) и касательных (б) составляющих вектора перемещений для угла ориентации $\alpha_{1}=48^{\circ}$

[Fig. 5. Distribution of the normal (a) and tangential (ס) components of displacement vector for orientation angle $\alpha_{1}=48^{\circ}$ ]

Анализ модели образования трещин в связующем волокнистого композита в процессе нагружения изгибающей нагрузкой сводится к параметрическому совместному исследованию объединенной разрешающей алгебраической системы задачи и критерия появления трещин (21) при различных значениях свободных параметров композитной пластины. Это различные геометрические и механические характеристики материалов связующего и армирующих волокон.

На некотором этапе нагружения пластины возможно одновременное существование в материале связующего зон пластического течения и образовавшихся трещин. Метод решения задачи теории изгиба пластины в этом случае объединяет одновременный учет повреждений и трещин с концевыми зонами пластических деформаций.

Выводы. Практика использования армированных волокнами композитов показывает, что на стадии проектирования следует принимать во внимание возможное появление в связующем трещин. Существующие методы прочностного расчета волокнистого композита, как правило, игнорируют это обстоятельство. Такое положение делает невозможным проектирование композита минимальной материалоемкости при гарантированной надежности и долговечности. Поэтому необходим предельный анализ композита, чтобы знать предельные изгибающие нагрузки, при которых в связующем происходит образование трещин. Размер предельных минимальных зон ослабленных межчастичных связей материала, при которых происходит трещинообразование, рекомендуется рассматривать как проектную характеристику материала связующего.

На основе предложенной расчетной модели, учитывающей в армированном волокнами композите наличие повреждений (зон ослабленных межчастичных связей материала), разработан метод расчета параметров композита, при которых появляются трещины. Зная основные значения предельных параметров формирования трещин и влияние на них свойств материалов, можно обоснованно управлять явлением образования трещин путем конструкторско-технологических решений на стадии проектирования композита.

(C) Гасанов Ш.Г., 2018

\section{Список литературы}

1. Мирсалимов В.М., Бахымов Ф.А. Обратная задача механики разрушения составной перфорированной пластины при изгибе // Проблемы машиностроения и надежности машин. 2005. № 5. С. 28-37.

2. Li S., Thouless M.D., Waas A.M., Schroeder J.A., Zavattieri P.D. Use of a cohesive-zone model to analyze the fracture of a fiber-reinforced polymer-matrix composite // Composites Science and Technology. 2005. Vol. 65. Pp. 537-549.

3. Бахышиов Ф.А., Мирсалимов В.М. Обратная двоякопериодическая задача теории изгиба пластины с упругими включениями // Прикладная механика и техническая физика. 2006. Т. 47. № 4. С. 153-161.

4. Lü N.C., Cheng Y.H., Si H.L., Cheng J. Dynamics of asymmetrical crack propagation in composite materials // Theoretical and Applied Fracture Mechanics. 2007. Vol. 47. Issue 3. Pp. 260-273. 
5. Мирсалимов В.M. Оптимальное проектирование составной пластины, ослабленной периодической системой трещин // Известия Российской академии наук. Механика твердого тела. 2007. № 1. С. 75-86.

6. Savastano Jr. H., Santos S.F., Radonjic M., Soboyejo W.O. Fracture and fatigue of natural fiber-reinforced cementitious composites // Cement and Concrete Composites. 2009. Vol. 31. Issue 4. Pp. 232-243.

7. Lü N., Li X., Cheng Y., Cheng J. An asymmetrical dynamic crack model of bridging fiber pull-out of composite materials. Fibers and Polymers. 2011. 12 (1). Pp. 79-88.

8. Ko Y.F., Ju J.W. Effects of fiber cracking on elastoplastic-damage behavior of fiber-reinforced metal matrix composites // Int. J. of Damage Mechanics. 2013. Vol. 22. Pp. 48-67.

9. Greco F., Leonetti L., Lonetti P. A two-scale failure analysis of composite materials in presence of fiber/matrix crack initiation and propagation // Composite Structures. 2013. Vol. 95. Pp. 582-597.

10. Mirsalimov V.M., Hasanov F.F. Interaction between periodic system of rigid inclusions and rectilinear cohesive cracks in an isotropic medium under transverse shear // Acta Polytechnica Hungarica. 2014. Vol. 11. Issue 5. Pp. 161-176.

11. Гасанов Ф.Ф. Разрушение композита, армированного однонаправленными волокнами // Механика композитных материалов. 2014. Т. 50. № 5. С. 829-842.

12. Мирсалимов В.М., Гасанов Ф.Ф. Взаимодействие периодической системы инородных упругих включений, поверхность которых равномерно покрыта однородной цилиндрической пленкой, и двух систем прямолинейных трещин с концевыми зонами // Проблемы машиностроения и надежности машин. 2014. Т. 43. № 5. C. $70-78$.

13. Hao W., Yao X., Ma Y., Yuan Y. Experimental study on interaction between matrix crack and fiber bundles using optical caustic method // Engineering Fracture Mechanics. 2015. Vol. 134. Pp. 354-367.

14. Гасанов Ф.Ф. Моделирование зарождения трещины сдвига в волокне композита, армированного однонаправленными волокнами // Проблемы машиностроения. 2014. Т. 17. № 2. С. 17-25.

15. Гасанов Ф.Ф. Зарождение трещин в изотропной среде с периодической системой круговых отверстий, заполненных жесткими включениями, при продольном сдвиге // Строительная механика инженерных конструкций и сооружений. 2014. № 3. С. 44-50.

16. Каюмов Р.А., Луканкин С.А., Паймушин В.Н. Идентификация механических характеристик армированных волокнами композитов // Ученые записки Казанского университета. Серия: Физико-математические науки. 2015. Т. 157. Кн. 4. С. 112-132.

17. Мирсалимов В.М., Гасанов Ф.Ф. Взаимодействие периодической системы инородных включений и когезионных трещин при продольном сдвиге // Строительная механика инженерных конструкций и сооружений. 2015. № 2. С. 18-28.

18. Полилов А.Н. Механизмы уменьшения концентрации напряжений в волокнистых композитах //
Прикладная механика и техническая физика. 2014. Т. 55. № 1. C. 187-197.

19. Мирсалимов В.М., Аскаров В.А. Минимизация параметров разрушения в композите при изгибе // Механика композитных материалов. 2015. Т. 51. № 6. C. $1049-1060$

20. Mokhtari A., Ouali M.O., Tala-Ighil N. Damage modelling in thermoplastic composites reinforced with natural fibres under compressive loading // Int. J. of Damage Mechanics. 2015. Vol. 24. Pp. 1239-1260.

21. Мирсалимов В.М., Аскаров В.А. Минимизация коэффициентов интенсивности напряжений для композита, армированного однонаправленными волокнами при изгибе // Вестник Чувашского государственного педагогического университета им. И.Я. Яковлева. Серия: Механика предельного состояния. 2016. № 3 (29). C. $105-116$.

22. Mirsalimov V.M., Hasanov F.F. Nucleation of cracks in an isotropic medium with periodic system of rigid inclusions under transverse shear // Acta Mechanica. 2015. Vol. 226. Pp. 385-395.

23. Круминьш Я., Зесерс А. Экспериментальное исследование разрушения бетона, армированного гибридными волокнами // Механика композитных материалов. 2015. Т. 51. № 1. С. 37-46.

24. Takeda T., Narita F. Fracture behavior and crack sensing capability of bonded carbon fiber composite joints with carbon nanotube-based polymer adhesive layer under Mode I loading // Composites Science and Technology. 2017. № 146. Pp. 26-33.

25. Ju J.W., $W u$ Y. Stochastic micromechanical damage modeling of progressive fiber breakage for longitudinal fiber-reinforced composites // Int. J. of Damage Mechanics. 2016. Vol. 25. Pp. 203-227.

26. Bakhshan H., Afrouzian A., Ahmadi H., Taghavimehr $M$. Progressive failure analysis of fiber-reinforced laminated composites containing a hole // Int. J. of Damage Mechanics. 2017. URL: https://doi.org/10.1177/105 6789517715088.

27. Mirsalimov V.M. Minimization of stress state of compound body weakened with cracks // Mechanics of Advanced Materials and Structures. 2018. URL: https://doi.org/10.1080/15376494.2018.1444220.

28. Мирсалимов В.М. Неодномерные упругопластические задачи. М.: Наука, 1987. 256 с.

29. Панасюк В.В. Механика квазихрупкого разрушения материалов. Киев: Наукова думка, 1991. 416 с.

30. Rusinko A., Rusinko K. Plasticity and creep of metals. Berlin: Springer, 2011. 434 p.

31. Мирсалимов В.M. Зарождение дефекта типа трещины во втулке контактной пары // Математическое моделирование. 2005. Т. 17. № 2. С. 35-45.

32. Мусхелишвили Н.И. Некоторые основные задачи математической теории упругости. М.: Наука, 1966. $707 \mathrm{c}$.

33. Панасюк В.В., Саврук М.П., Даџьшиин А.П. Распределение напряжений около трещин в пластинах и оболочках. Киев: Наукова думка, 1976. 443 с.

34. Саврук М.П. Двумерные задачи упругости для тел с трещинами. Киев: Наукова думка, 1981. 324 с. 


\section{References}

1. Mirsalimov V.M., Bakhyshov F.A. (2005). Inverse problem of the fracture mechanics of a composite perforated plate during bending. Journal of Machinery Manufacture and Reliability, 34(5), 28-37.

2. Li S., Thouless M.D., Waas A.M., Schroeder J.A., Zavattieri P.D. (2005). Use of a cohesive-zone model to analyze the fracture of a fiber-reinforced polymer-matrix composite. Composites Science and Technology, 65, 537-549.

3. Bakhyshov F.A., Mirsalimov V.M. (2006). Inverse doubly periodic problem of the theory of bending of a plate with elastic inclusions. Journal of Applied Mechanics and Technical Physics, 47(4), 588-595.

4. Lü N.C., Cheng Y.H., Si H.L., Cheng J. (2007). Dynamics of asymmetrical crack propagation in composite materials. Theoretical and Applied Fracture Mechanics, 47(3), 260-273.

5. Mirsalimov V.M. (2007). Optimal design of a compound plate weakened by a periodic crack system. Mechanics of Solids, 42(2), 231-240.

6. Savastano Jr H., Santos S.F., Radonjic M., Soboyejo W.O. (2009). Fracture and fatigue of natural fiber-reinforced cementitious composites. Cement and Concrete Composites, 31, 232-243.

7. Lü N., Li X., Cheng Y., Cheng J. (2011). An asymmetrical dynamic crack model of bridging fiber pull-out of composite materials. Fibers and Polymers, 12(1), 79-88.

8. Ko Y.F., Ju J.W. (2013). Effects of fiber cracking on elastoplastic-damage behavior of fiber-reinforced metal matrix composites. International Journal of Damage Mechanics, 22, 48-67.

9. Greco F., Leonetti L., Lonetti P. A two-scale failure analysis of composite materials in presence of fiber/ matrix crack initiation and propagation. Composite Structures, 2013, 95, 582-597.

10. Mirsalimov V.M., Hasanov F.F. (2014). Interaction between periodic system of rigid inclusions and rectilinear cohesive cracks in an isotropic medium under transverse shear. Acta Polytechnica Hungarica, 11(5), 161-176.

11. Hasanov F.F. (2014). Fracture of a composite reinforced by unidirectional fibers. Mechanics of Composite Materials, 50(5), 593-602.

12. Mirsalimov V.M., Hasanov F.F. (2014). Interaction of a periodic system of foreign elastic inclusions whose surface is uniformly covered with a homogeneous cylindrical film and two systems of straight line cracks with end zones. Journal of Machinery Manufacture and Reliability, 43(5), 408-415.

13. Hao W., Yao X., Ma Y., Yuan Y. (2015). Experimental study on interaction between matrix crack and fiber bundles using optical caustic method. Engineering Fracture Mechanics, 134, 354-367.

14. Hasanov F.F. (2014). Modelling of crack nucleation in the fibre of composite reinforced with unidirectional fibres under shear. Journal of Mechanical Engineering, 17(2), 17-25. (In Russ.)

15. Hasanov F.F. (2014). Nucleation of cracks in isotropic medium with periodic system of the circular holes filled with rigid inclusions at longitudinal shear.
Structural Mechanics of Engineering Constructions and Buildings, (3), 44-50. (In Russ.)

16. Kayumov R.A., Lukankin S.A., Paimushin V.N., Kholmogorov S.A. (2015). Identification of mechanical properties of fiber-reinforced composites. Proceedings of Kazan University. Physics and Mathematics Series, 157(4), 112-132. (In Russ.)

17. Mirsalimov V.M., Hasanov F.F. (2015). Interaction of periodic system heterogeneous inclusions and cohesive cracks under longitudinal shear. Structural Mechanics of Engineering Constructions and Buildings, (2), 18-28. (In Russ.)

18. Polilov A.N. (2014). Mechanisms of stress concentration reduction in fiber composites. Journal of Applied Mechanics and Technical Physics, 55(1), 154-163.

19. Mirsalimov V.M., Askarov V.A. (2016). Minimization of fracture parameters of a composite at bending. Mechanics of Composite Materials, 51(6), 737-744.

20. Mokhtari A., Ouali M.O., Tala-Ighil N. (2015). Damage modelling in thermoplastic composites reinforced with natural fibres under compressive loading. International Journal of Damage Mechanics, 2015, 24, 1239-1260.

21. Mirsalimov V.M., Askarov V.A. (2016). Minimization of stress intensity factors for composite reinforced by unidirectional fibers at bending. I. Yakovlev Chuvash State Pedagogical University Bulletin. Series. Mechanics of a Limit State, 3(29), 105-116. (In Russ.)

22. Mirsalimov V.M., Hasanov F.F. (2015). Nucleation of cracks in an isotropic medium with periodic system of rigid inclusions under transverse shear. Acta Mechanica, 226, 385-395.

23. Krūminsš J., Zesers A. (2015). Experimental investigation of the fracture of hybrid-fiber-reinforced concrete. Mechanics of Composite Materials, 51(1), 25-32.

24. Takeda T., Narita F. (2017). Fracture behavior and crack sensing capability of bonded carbon fiber composite joints with carbon nanotube-based polymer adhesive layer under Mode I loading. Composites Science and Technology, 146, 26-33.

25. Ju J.W., Wu Y. (2016). Stochastic micromechanical damage modeling of progressive fiber breakage for longitudinal fiber-reinforced composites. International Journal of Damage Mechanics, 25, 203-227.

26. Bakhshan H., Afrouzian A., Ahmadi H., Taghavimehr M. (2017). Progressive failure analysis of fiberreinforced laminated composites containing a hole. International Journal of Damage Mechanics, https://doi.org/ $10.1177 / 1056789517715088$.

27. Mirsalimov V.M. (2018). Minimization of stress state of compound body weakened with cracks. Mechanics of Advanced Materials and Structures, https://doi.org/ 10.1080/15376494.2018.1444220.

28. Mirsalimov V.M. (1987). Neodnomernye Uprugoplasticheskie Zadachi [Non-one-dimensional Elastoplastic Problems]. Moscow, Nauka, 256. (In Russ.)

29. Panasyuk V.V. (1991). Mehanika Kvazihrupkogo Razrushenija Materialov [Mechanics of Quasibrittle Fracture of Materials]. Kiev, Naukova Dumka, 416. (In Russ.)

30. Rusinko A., Rusinko K. (2011). Plasticity and Creep of Metals. Berlin; Springer, 434. 
31. Mirsalimov V.M. (2005). Nucleation of crack type defect in the hub of a contact pair. Matematicheskoe Modelirovanie, 17(2), 35-45.

32. Muskhelishvili N.I. (1977). Some Basic Problem of Mathematical Theory of Elasticity. Amsterdam: Kluwer Academic, 707.

33. Panasyuk V.V., Savruk M.P., Datsyshyn A.P. (1976). Raspredelenie Naprjazhenij okolo Treshhin v Plastinah $i$ Obolochkah [The Stress Distribution around Cracks in Plates and Shells]. Kiev, Naukova Dumka, 443. (In Russ.)

34. Savruk M.P. (1981). Dvumernye Zadachi Uprugosti dlja Tel s Treshhinami [Two-dimensional Problem of Elasticity for Bodies with Cracks]. Kiev, Naukova Dumka, 324. (In Russ.)

\section{Об авторе}

Гасанов Шахин Гумбат огль - доктор технических наук, профессор кафедры организации автомобильных перевозок и дорожного движения, Азербайджанский технический университет. Научные интере$c b l$ : прочность дорожных покрытий, механика разрушения конструкций и сооружений. Контактная информация: e-mail - hssh3883@gmail.com.

\section{About the author}

Shahin H. Hasanov - Doctor of Technical Sciences, Professor, Professor of the Automobile Transportation and Organization of Transport Department, Azerbaijan Technical University. Scientific interests: strength of road covering, fracture mechanics of structures and constructions. Contact: e-mail-hssh3883@gmail.com.

\section{Для цитирования}

Гасанов Ш.Г. Моделирование трещинообразования в волокнистом композите при изгибе // Строительная механика инженерных конструкций и сооружений. 2018. T. 14. № 3. C. 248-257. DOI: $10.22363 / 1815-5235-2018-$ $14-3-248-257$.

\section{For citation}

Hasanov SH.H. (2018). Modeling of cracks nucleation in fiber composite under bending. Structural Mechanics of Engineering Constructions and Buildings. 14(3), 248-257. DOI: 10.22363/1815-5235-2018-14-3-248-257. 